\title{
Fabrication of a microarray using a combination of the large circular sense and antisense DNA
}

\author{
KYUNG-OH DOH ${ }^{1 *}$, YUN-HAN LEE ${ }^{2 *}$, KIL-HWAN HAN ${ }^{2 *}$, SEOK-YONG UHM ${ }^{2}$, JONG-PIL KIM ${ }^{2}$, \\ YUN-UI BAE ${ }^{1}$, JEONG-HOH PARK ${ }^{2}$, IK-JAE MOON ${ }^{2}$ and JONG-GU PARK ${ }^{2,3,4}$ \\ ${ }^{1}$ Department of Physiology, Yeungnam University School of Medicine, 317-1 Daemyung-dong, Daegu 705-717; \\ ${ }^{2}$ WelGENE Inc., 71B-4L, Hightech Sector 2, Sungseo Industrial Park 3, Dalseogu, Daegu 704-230; \\ ${ }^{3}$ Department of Medical Genetic Engineering, and ${ }^{4}$ Chronic Disease Research Center and Institute \\ for Medical Science, Keimyung University School of Medicine, Dongsan Medical Center, \\ 194 Dongsan-dong, Joong-gu, Daegu 700-712, Korea
}

Received August 6, 2009; Accepted October 16, 2009

DOI: 10.3892/ijmm_00000320

\begin{abstract}
In the present study, single-stranded large circular (LC)-sense molecules were utilized as probes for DNA microarrays and showed stronger binding signals than those of PCR-amplified cDNA probes. A microarray experiment using 284 LC-sense DNA probes found 6 upregulated and 7 downregulated genes in A549 cells as compared to WI38VA13 cells. Repeated experiments showed largely consistent results, and microarray data strongly correlated with data acquired from quantitative real-time RT-PCR. A large array comprising 5,079 LC-sense DNA was prepared, and analysis of the mean differential expression from dye-swap experiments revealed 332 upregulated and 509 downregulated genes in A549 cells compared to WI38VA13 cells. Subsequent functional analysis using an LC-antisense library of overexpressed genes identified 28 genes involved in A549 cell growth. These experiments demonstrated the proper features of LC-sense molecules as probe DNA for microarray and the potential utility of the combination of LC-sense and -antisense libraries for an effective functional validation of genes.
\end{abstract}

\section{Introduction}

Recent developments in DNA microarray technologies have given us the chance to study the relative expression levels of

Correspondence to: Dr Jong-Gu Park, Department of Medical Genetic Engineering, Keimyung University School of Medicine, 194 Dongsan-dong, Joong-gu, Daegu 700-712, Korea

Dr Kyung-Oh Doh, Department of Physiology, Yeungnam University School of Medicine, 317-1 Daemyung-dong, Daegu 705-717, Korea

E-mail: jonggu@dsmc.or.kr; kodoh@ynu.ac.kr

${ }^{*}$ Contributed equally

Key words: microarray, expression profiling, antisense, lung cancer a large number of genes, in a massively parallel mode (1-4). Using array hybridization techniques, the expression of thousands or tens of thousands of genes can be utilized in a variety of applications (5-8). This rapid accumulation of genomic sequence information and expression profiling has created a bottleneck in subsequent target validation for diverse applications including drug discovery. The majority of target gene validation via functional studies has been conducted using a variety of conventional gain-of-function or loss-offunction studies. These loss-of-function studies have been conducted either by gene knockdown using conventional antisense $(9,10)$, decoy $(11)$, ribozymes $(12)$, or more recently small-interfering RNA (siRNA) (13-17).

Although microarrays now play an important role in biomedical sciences, inconsistencies or errors in the microarray data originate from a variety of sources including crosshybridization, sequencing mistakes, contamination of clones and uneven hybridization conditions across an array (18). The signal intensity of the microarray data is thought to be another important factor for interpretation of experimental results (19). In microarray experiments employing doublestranded DNA as probes, the probe DNA requires denaturation in order to bind a labeled cDNA. Whereas denatured DNAs generate weaker binding signals due to the renaturation of the parental complementary strands, single-stranded DNA generates a stronger binding signal. In fact, single-stranded DNA was previously employed as probe DNA for microarray experiments at the Sanger Institute (Cambridge, UK). The M13 phagemid, a recombinant plasmid vector which is used for the construction of recombinant bacteriophages, can be engineered to generate a large quantity of single-stranded large circular (LC) molecules harboring either the sense or antisense sequence of a gene. Recently, high-throughput functional genomics using LC-antisense molecules has been developed for the identification of genes associated with cancer cell growth (20). The LC-sense DNA of recombinant bacteriophages may result in advantages for higher chances of binding to complementary target cDNA, owing to its considerable length and high degree of sequence fidelity. LC-sense DNA can also be easily generated in a high- 
throughput and large-scale mode in transformed E. coli cultures.

In the present study, the utility of LC-sense molecules instead of LC-antisense was examined for the fabrication of a DNA microarray for the enhanced detection of differential gene expression. The data reproducibility of the LC-sense DNA microarray was further tested by employing quantitative real-time RT-PCR. An LC-antisense library to upregulated genes was next constructed and used in the functional validation of genes associated with the growth of cancer cells.

\section{Materials and methods}

Cell culture and RNA preparation. Both the human lung cancer cell line A549 and oncogenic transformants of human lung embryonic fibroblasts WI38VA13 were acquired from the Korean Cell Line Bank and cultured in DMEM and RPMI media, respectively, containing $10 \%$ fetal bovine serum (WelGENE, Daugu, Korea). All cultures were maintained in an atmosphere containing $5 \% \mathrm{CO}_{2}$ and harvested for total RNA isolation. RNA was used only in cases in which the OD 260/ 280 ratio was $>1.8$.

High-throughput preparation of LC-sense DNA probes in a large quantity. Either pSPORT1 or pT3T7-Pac phagemids, harboring human EST cDNA, were utilized in the production of single-stranded phage genomic DNA harboring the sense cDNA sequences. The recombinant phagemids were transformed into competent $E$. coli cells, XL-1 Blue (Stratagene, La Jolla, CA, USA), which had been infected with the helper bacteriophage, M13K07 (New England Biolabs, Ipswich, MA, USA), in accordance with a previously described method $(20,21)$. All of the LC-sense DNA was adjusted to a uniform concentration of $0.35 \mathrm{~g} / \mathrm{l}$.

Microarray preparation, target cDNA labeling and hybridization. The microarrays were prepared via the spotting of the LC-sense probe DNA onto poly-l-lysine glass slides using an Affymetrix 417 Arrayer (Affymetrix, Inc., Santa Clara, CA, USA). In addition, LC-sense molecules to 4 housekeeping genes (glyceraldehyde 3-phosphate dehydrogenase, phospholipase A2, tubulin- $\alpha 2$ and $B$-actin) were purified as internal controls, and 3 different vector molecules [pSPORT1, pT3T7-Pac and pBluescript KS(+) phagemids] were purified as negative controls. IntelliGene Human Cancer Chip, ver. 4.0, comprised of the PCR-amplified cDNA probes of 886 cancerrelated genes, was purchased from Takara Bio (Kyoto, Japan). Total RNA samples $(30 \mu \mathrm{g})$ from A549 and WI38VA13 cells were reverse-transcribed with oligo-dT primers in the presence of Cy5-dUTP and Cy3-dUTP, respectively. Hybridization was conducted at $66^{\circ} \mathrm{C}$ for $16 \mathrm{~h}$ in a humidified chamber. After hybridization, the slides were washed once each in $2 \mathrm{X} \mathrm{SSC} / 0.2 \% \mathrm{SDS}$ for $5 \mathrm{~min}$ at $55^{\circ} \mathrm{C}$, $2 \mathrm{X} \mathrm{SSC} / 0.2 \% \mathrm{SDS}$ for $5 \mathrm{~min}$ at $65^{\circ} \mathrm{C}, 2 \mathrm{X} \mathrm{SSC}$ for $5 \mathrm{~min}$ at room temperature, and $0.05 \mathrm{X} \mathrm{SSC}$ for $5 \mathrm{~min}$ at room temperature, then spin-dried in a centrifuge prior to image scanning.

Microarray data acquisition and analysis. The fluorescent target cDNAs were detected by scanning the slides with an
Affymetrix 428 Array Scanner (Affymetrix, Inc.) or GenePix 4000B (Molecular Devices, Sunnyvale, CA, USA). The scanned images were then analyzed with ImaGene ver. 4.2 software (BioDiscovery, El Segundo, CA, USA). The signal intensity values were determined by subtracting the background from the median values of the intensity of the spots. The corrected background intensities were then normalized to total signal intensities in order to account for the different input RNA concentrations or labeling efficiency in the individual $\mathrm{Cy} 3$ and $\mathrm{Cy} 5$ reverse transcriptase reactions. The microarray data (GSE11278) were deposited in Gene Expression Omnibus (GEO).

Quantitative real-time $R T$-PCR. Total RNA ( $1 \mu \mathrm{g})$ was reverse-transcribed using the random primers provided in the Reverse Transcription System (Promega, Madison, WI, USA). The cDNAs of the target genes were amplified using the Dynamo HS SYBR Green qPCR Kit (Finzymes, Espoo, Finland) and the DNA Engine Opticon 2 System (MJ Research, Waltham, MA, USA). In order to normalize the quantity of total RNA in each reaction, the $\beta$-actin gene was simultaneously amplified.

Transfection of an LC-antisense library into a lung cancer cell line. An LC-antisense library to upregulated genes in the $5 \mathrm{~K}$ LC-sense DNA microarray experiments was constructed by unidirectional cloning and transfected into A549 cells. The cells $\left(1 \times 10^{3}\right)$ were then seeded in each well of 96 -well plates in $100 \mu 1$ of DMEM media supplemented with $10 \%$ FBS. The cells were incubated for $12-18 \mathrm{~h}$ at $37^{\circ} \mathrm{C}$ in a $5 \% \mathrm{CO}_{2}$ incubator. The LC-antisense library $(0.1 \mu \mathrm{g})$ complexed with $0.3 \mu \mathrm{g}$ of Enhancer Q and $0.5 \mu \mathrm{g}$ of WelFect Ex (WelGENE) was added to the cultured cells and incubated for 3 additional days. Equal quantities of control LC-antisense DNA lacking a cDNA insert complexed with transfection reagents were also added to the same number of cells in different 96-well plates and simultaneously assayed. After transfection, microscopic observations and MTT reduction assays were conducted. The percentage of the inhibition of cell growth in each well treated with LC-antisense was calculated via the comparison of the optical density with those of the sham treatments.

\section{Results}

Production of LC-sense DNA as microarray probes. The recombinant M13 phagemid was employed in the production of single-stranded LC-sense DNA molecules. We utilized these molecules as probe DNA for the DNA microarray trials. LCsense molecules were generated as genomic DNA using the F1 replication origin of the phagemid vector. The recombinant phagemids were transformed into competent $E$. coli infected with the helper phage, M13K07. From $100 \mathrm{ml}$ of the culture supernatant, an approximate quantity of $150 \mu \mathrm{g}$ LC-sense probe DNA was acquired.

Stronger binding signal of LC-sense probe DNA to target cDNAs. In order to compare the signal sensitivity of LCsense DNA to that of conventional PCR-amplified cDNA in the probe-target interaction, we assessed the signal intensities 
obtained under identical hybridization conditions. The differences in the signal intensities between the two types of probes were further studied via serial dilution, using a probe to verify the fold difference of the signal intensities. Both LC-sense and PCR-amplified cDNA probes to the gene of the serine protease inhibitor, Kunitz type 2 (SPINT2) were prepared at concentrations of $0.35 \mathrm{~g} / \mathrm{l}$. Whereas the PCR-amplified cDNA probes were spotted at the original concentration, the LC-sense DNA probes were successively diluted from the original concentration by factors of 2-, 5-, 10- and 20-fold (Fig. 1A). The probe samples were then spotted alongside PCR-amplified cDNA probes of $B$-actin (positive control) and Lambda A (negative control) genes on poly-1-lysine-coated glass slides and were quality-controlled by examining the uniform spotting in a quantitative manner. When signals from the denatured cDNA probes were compared to those from the LC-sense DNA probes, the median signal intensity generated from the 5 -fold dilution of the LC-sense DNA was found to be somewhat higher than those from the PCR-amplified cDNA probes (Fig. 1B). Subsequently, the LC-sense probe DNA to 284 cancer-associated genes was generated to fabricate a DNA microarray. Differential expression profiles between WI38VA13 and A549 cells were evaluated with Cy3-dUTP and Cy5-dUTP labeling of total RNA from respective cells. The LC-sense DNA microarray and cDNA microarray (IntelliGene Cancer DNA Chip, Takara Bio) were hybridized and scanned. Data of the 204 genes shared by the LC-sense DNA microarray and cDNA microarray were selectively scatter-plotted after $\log _{2}$ transformation (Fig. 1C and D). The scatter plot obtained from the LC-sense DNA microarray showed significantly stronger signals and tighter clustering along the $y=x$ line for most values compared with those from the cDNA microarray. The median signal intensities of $\mathrm{Cy} 3-$ and Cy5-labeled target cDNAs on the LC-sense DNA microarray were 4.2- and 12.5-fold higher, respectively, than those of the cDNA microarray.

Reproducibility in gene expression profiling with LC-sense probe DNA. In the microarray experiment using LC-sense DNA, 6 of the $284(\sim 2.1 \%)$ genes were upregulated $>2$-fold in A549 cells. Of these 6 genes, the CatL (22), GPCR19 (23), JAG1 (24), K8 (25) and SPINT2 (26) genes were previously reported as being involved in the progression of lung cancer. Conversely, 7 of the $284(\sim 2.5 \%)$ genes were downregulated $>2$-fold. Among these 7 genes, the CTGF (27), LAMB2 (28) and tPA (29) genes were previously reported to be downregulated in lung cancer. In addition, the downregulation of the CDH11 gene was detected in astrocytoma cells (30). When the experiments were repeated three more times to determine the reproducibility of the LC-sense DNA, the patterns of upregulation or downregulation of the genes were determined to be largely consistent, with only a minor degree of discordance (Table I).

Validation of LC-sense DNA microarray data with quantitative real-time $R T$-PCR. The differential expression profiles acquired from the LC-sense DNA microarray platform were then evaluated by real-time RT-PCR for data validation. Thirteen genes were determined to have been either upregulated or downregulated in A549 cells from the
A

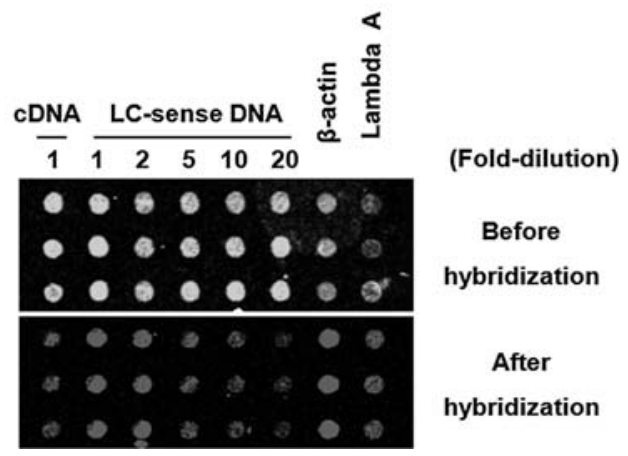

B

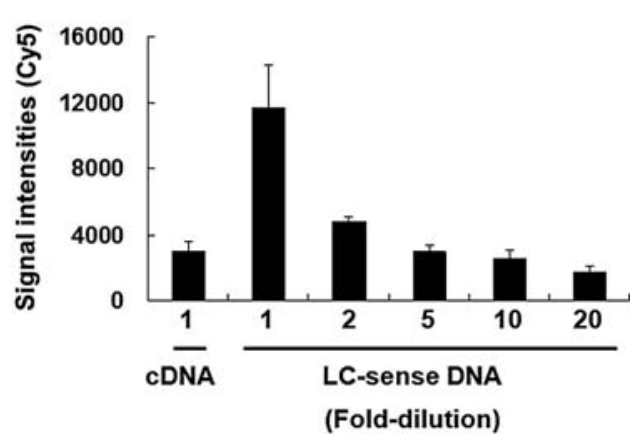

C

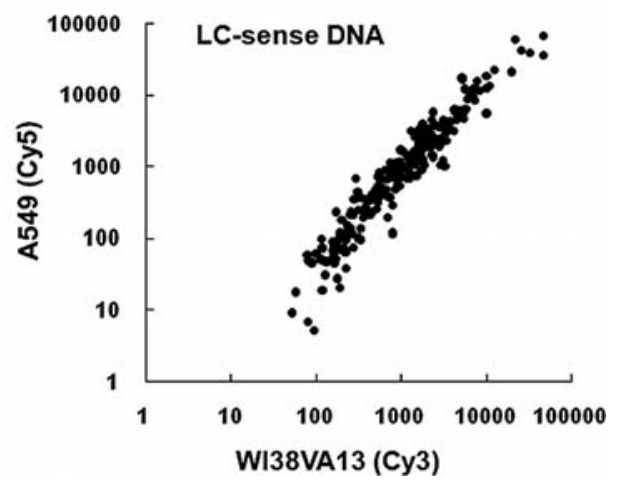

D

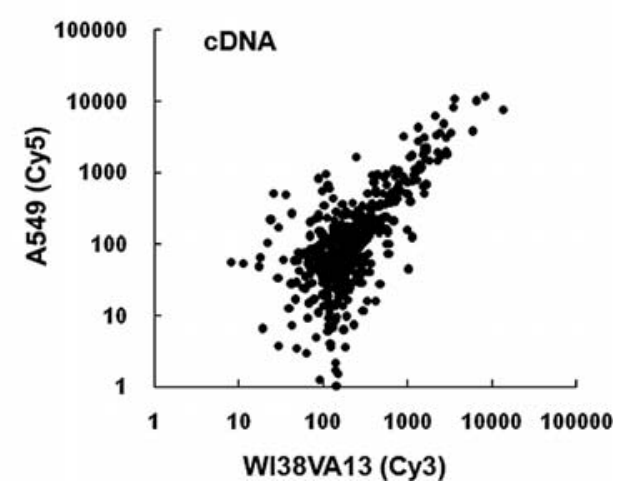

Figure 1. The fold difference in hybridization signals and microarray experiments between the LC-sense DNA and the PCR-amplified cDNA. (A) After confirming the uniform spotting of the probe DNA, the microarray slides were hybridized with Cy5-labeled target cDNAs of A549 cells. (B) The signal intensity of three spots is shown in a bar graph as the mean \pm SD. The numbers shown underneath the graph denote the fold dilutions of the probe DNA. (C and D) The labeled target cDNAs from the WI38VA13 (Cy3) and A549 (Cy5) cells were mixed together and hybridized to an LC-sense DNA microarray and cDNA microarray. Each microarray was scanned and scatter-plotted after $\log _{2}$ transformation. 
Table I. Upregulated or downregulated genes in the lung cancer cell line A549.

Gene description

Accession

no. $\mathrm{Cy} 5 / \mathrm{Cy} 3$
ratio $^{\mathrm{a}}$
Up-regulated genes in A549

Cathepsin L (CatL)

G protein-coupled receptor 19 (GPCR19)

Jagged 1 (JAG1)

Keratin 8 (K8)

Serine protease inhibitor, Kunitz type 2 (tissue factor pathway inhibitor-2, TFPI2)

Thioredoxin reductase 1 (TrxR1)

Down-regulated genes in A549

Cadherin 11, type 2, OB-cadherin (osteoblast) (CDH11)

Collagen, type VI, a 3 (COL6A3)

Connective tissue growth factor (CTGF)

Cyclin-dependent kinase inhibitor 2C (p18, inhibits CDK4) (CDKN2C)

Interferon induced transmembrane protein 2 (1-8D) (IFITM2)

Laminin, B 2 (laminin S) (LAMB2)

Tissue plasminogen activator (tPA)

$\begin{array}{llll}\text { AI814383 } & 2.18 & 0.10 & 4 / 4 \\ \text { H07878 } & 2.27 & 0.27 & 4 / 4 \\ \text { NM000214 } & 3.24 & 1.06 & 3 / 4 \\ \text { AA598517 } & 5.96 & 0.38 & 4 / 4 \\ \text { NM021102 } & 4.15 & 0.21 & 4 / 4 \\ \text { AA464849 } & 2.93 & 1.12 & 3 / 4 \\ & & & \\ \text { AA136983 } & 0.43 & 0.07 & 4 / 4 \\ \text { R62603 } & 0.36 & 0.10 & 4 / 4 \\ \text { AA59874 } & 0.32 & 0.05 & 4 / 4 \\ \text { N72115 } & 0.43 & 0.08 & 4 / 4 \\ \text { AA862371 } & 0.57 & 0.11 & 3 / 4 \\ \text { AA156802 } & 0.48 & 0.03 & 4 / 4 \\ \text { AA447797 } & 0.42 & 0.04 & 4 / 4\end{array}$

All genes listed showed at least a 2-fold upregulation or downregulation as compared to the expression level in the WI38VA13 cells on the LCsense DNA microarray in at least three out of four independent experiments. ${ }^{a}$ Each data point represents the average of the repeated experiments. ${ }^{\mathrm{b}}$ The frequency by which gene expression was upregulated at least 2-fold in the repetitive experiments $(\mathrm{k} / \mathrm{n})$ is shown. SD, standard deviation.

A

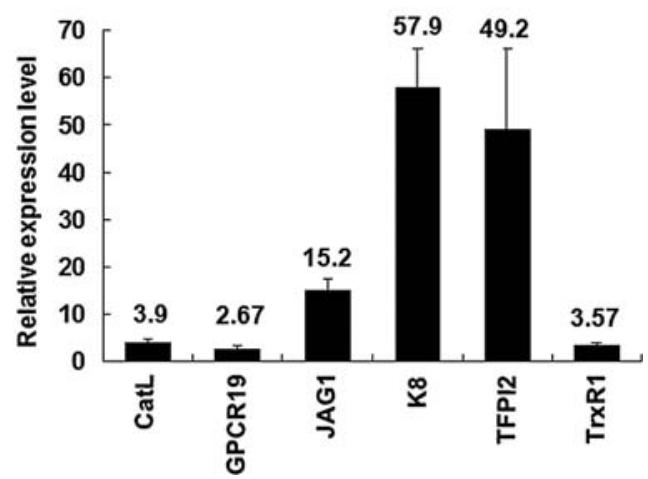

B

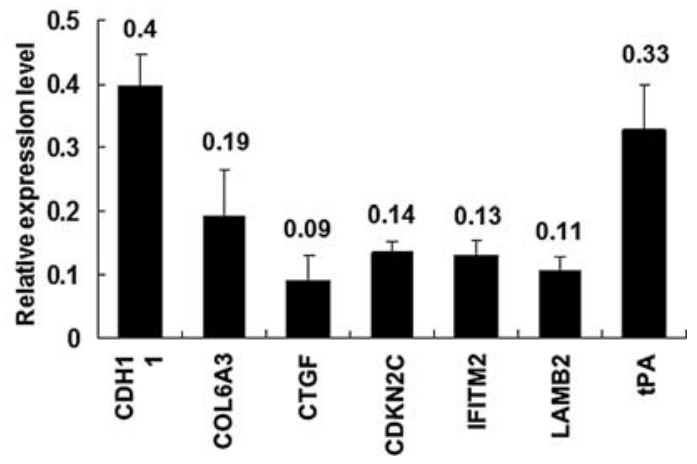

Figure 2. Validation of LC-sense DNA microarray data. The reliability of microarray signals was examined for 6 upregulated genes (A) and 7 downregulated genes (B) by quantitative analysis using real-time RT-PCR in A549 cells. The expression levels were calculated relative to that of B-actin that had been normalized to 1.0 for WI38VA13 cells. Values of differential expression represent the average and SD (bars) of three independent experiments. Genes with differential expression are shown underneath each figure.

microarray experiments. The expression levels of the genes in A549 cells were quantified relative to those from the WI38VA13 cells. The expression levels of the CatL, GPCR19, JAG1, K8, SPINT2 and TrxR1 genes in A549 cells were higher than those observed in the WI38VA13 cells (Fig. 2A). Meanwhile, the expression levels of the CDH11, COL6A3, CTGF, CDKN2C, IFITM2, LAMB2 and tPA genes in A549 were lower when compared to levels observed in the WI38VA13 cells (Fig. 2B). In contrast, the expression levels of the GLB1 (galactosidase B 1) and HDAC1 (histone deacetylase 1) genes, which showed almost no changes in the LC-sense DNA microarray experiment, were not signifi- cantly altered. Furthermore, fold-changes between the expression levels measured using real-time RT-PCR and those using LC-sense DNA microarray exhibited a correlation coefficiency of $\mathrm{R}^{2}=0.9189$.

Expression profiling with $5 K$ LC-sense DNA microarray. In order to verify the utility of the system for large scale expression profiling, LC-sense DNA probes to 5,079 human unigene clones were generated and arrayed on glass slides. The 5K LC-sense DNA microarray was then hybridized with a mixture of labeled target cDNAs from A549 and WI38VA13 cells. The microarray was then scanned (Fig. 3A). A dye- 
A

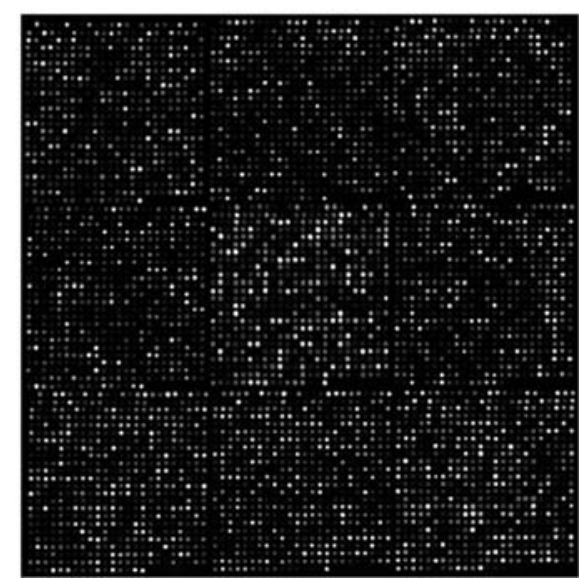

B

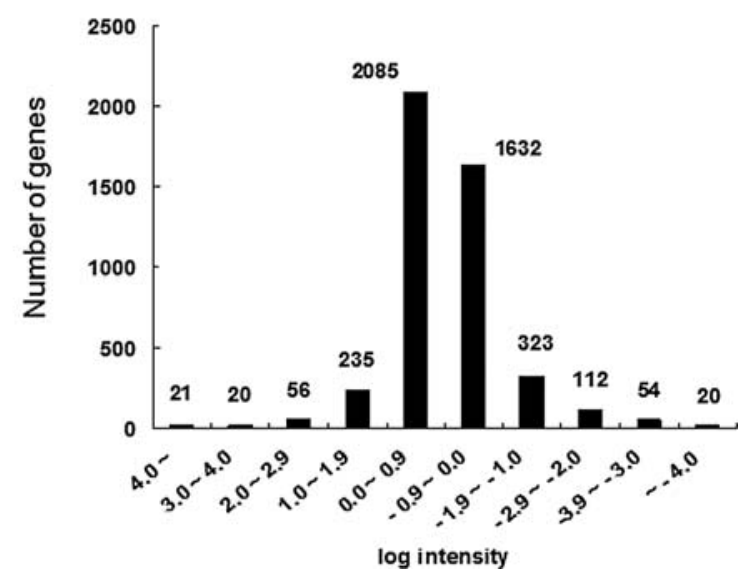

c

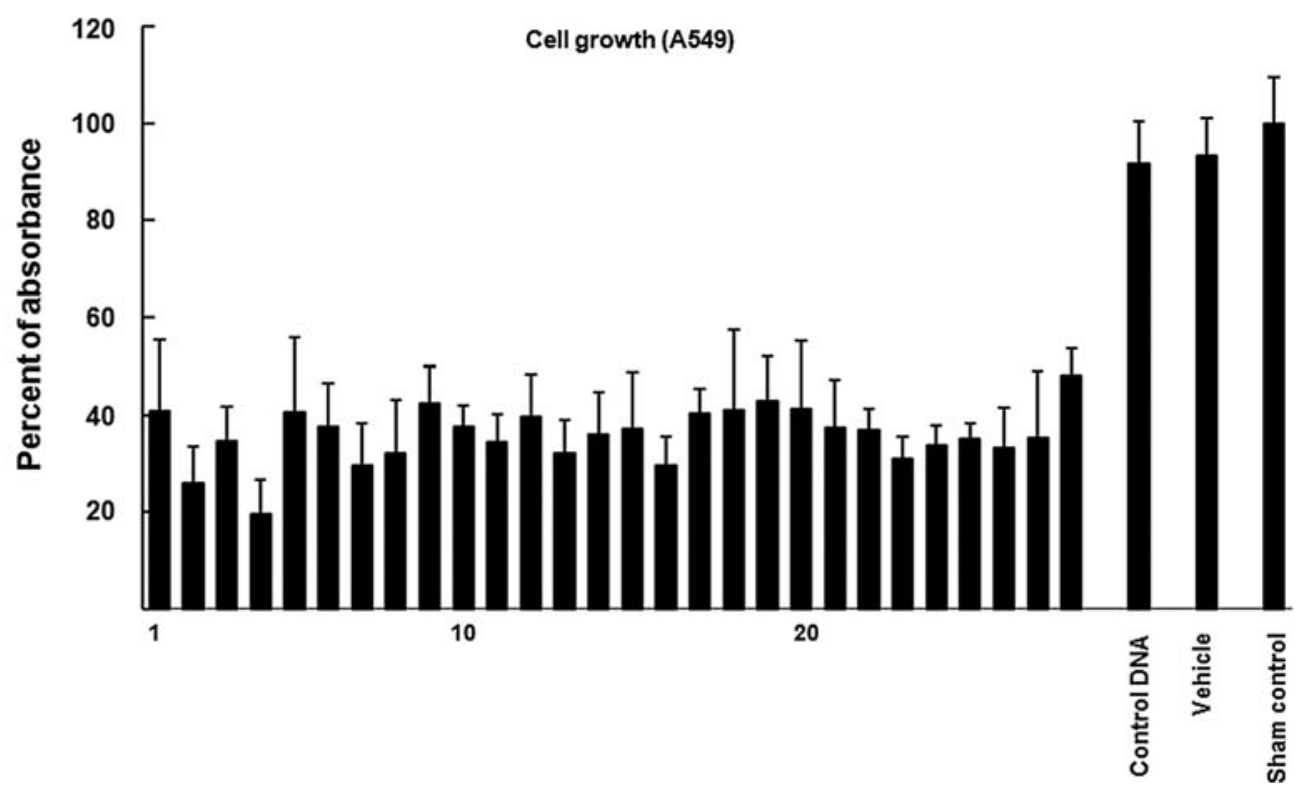

Figure 3. Large-scale expression profiling in A549 cells using a 5K LC-sense DNA microarray. (A) LC-sense DNA probes to 5,079 human unigenes were prepared and arrayed on a glass slide. The microarray slide was preheated and hybridized with a mixture of labeled target cDNAs. The fluorescence image of the microarray was scanned. (B) The number of genes for each expression range from dye-swap microarray experiments is shown on the ordinate of the graph. (C) LC-antisense species of 360 genes were transfected into A549 cells, and the growth rates were assessed by MTT assays in triplicate. Twenty-eight members of the LC-antisenses from the LC-antisense library showed marked inhibition (survival <50\%) of growth in A549 cells as compared to the sham control, while these did not significantly inhibit (survival $>80 \%$ ) the growth of WI38VA13 cells. The value of each bar represents the mean \pm SD of triplicate experiments.

swap test was conducted for quality control, and showed a correlation coefficiency of $\mathrm{R}^{2}=0.9351$. A histogram of differential gene expression was also generated (Fig. 3B). Analysis of the mean differential expression levels by $\log$ transformation from the dye-swap microarray experiments showed that 332 $(6.54 \%)$ of $5 \mathrm{~K}$ were upregulated $>2$-fold, and $509(10.02 \%)$ out of $5 \mathrm{~K}$ were downregulated $>2$-fold, respectively, in A549 cells.

Functional validation of target genes using LC-antisense library. The LC-antisense library to the genes upregulated in A549 cells was constructed and utilized in the identification of genes functionally involved in the growth of lung cancer cells.
Three hundred and sixty overexpressed genes selected from preliminary microarray experiments were unidirectionally subcloned into pBluescript SK (-) phagemids. These recombinant phagemids were then independently transformed into competent $E$. coli cells, and LC-antisense molecules were then purified. The transfected cells were quantitatively measured for growth inhibition via MTT assay 3 days after transfection. We tried to select the targets which have strong inhibitory effects on the growth of A549 cells with minimal effects on the growth of WI38VA13 cells. Twenty-eight members (Table II) of 360 antisenses showed marked inhibitory effects (survival $<50 \%$ ) on the growth of A549 (Fig. 3C) compared to WI38VA13 cells, while showing minimal effects (survival 
Table II. Twenty-eight LC-antisenses which showed marked inhibition of growth in A549 as compared to WI38VA13 cells.

\begin{tabular}{|c|c|c|}
\hline $\begin{array}{l}\text { Representative } \\
\text { RNA }\end{array}$ & Symbol & Description \\
\hline AA700054 & ADFP & Adipose differentiation-related protein \\
\hline AA774619 & ATP5SL & Hypothetical protein FLJ10241 \\
\hline NM_005603.3 & ATP8B1 & ATPase, class I, type 8B, member 1 , \\
\hline AA026120 & BHLHB2 & Basic helix-loop-helix domain containing, class B, 2 \\
\hline AA426053 & CAPN3 & Calpain 3, (p94) \\
\hline AA430524 & CAPZB & Capping protein (actin filament) muscle Z-line, $B$ \\
\hline AA490911 & CD99L2 & CD99 antigen-like 2 \\
\hline AA877213 & CYP24A1 & Cytochrome P450, family 24 , subfamily A, polypeptide 1 \\
\hline AA149095 & DUSP1 & Dual specificity phosphatase 1 \\
\hline R61289 & L3MBTL & L(3)mbt-like (Drosophila) \\
\hline AA625666 & LITAF & Lipopolysaccharide-induced TNF factor \\
\hline AA702487 & LY6G5C & Lymphocyte antigen 6 complex, locus G5C \\
\hline R83837 & LYN & V-yes-1 Yamaguchi sarcoma viral-related oncogene homolog \\
\hline NM_007289.1 & MME & $\begin{array}{l}\text { Membrane metallo-endopeptidase (neutral endopeptidase, enkephalinase, CALLA, CD10), } \\
\text { transcript variant } 2 b\end{array}$ \\
\hline AA894577 & NOL5A & Nucleolar protein 5A (56 kDa with KKE/D repeat) \\
\hline H61726 & NR1H3 & Nuclear receptor subfamily 1 , group $\mathrm{H}$, member 3 \\
\hline AI739498 & PPARA & Peroxisome proliferative activated receptor, $\alpha$ \\
\hline AA282134 & QPCT & Glutaminyl-peptide cyclotransferase (glutaminyl cyclase) \\
\hline NM_006808.2 & SEC61B & Sec61 ß subunit (SEC61B) \\
\hline AA454570 & SEMA3F & Sema domain, immunoglobulin domain (Ig), short basic domain, secreted, (semaphorin) 3F \\
\hline N47445 & SFRP4 & Ependymin related protein 1 (zebrafish) \\
\hline AA402891 & SLC29A2 & Solute carrier family 29 (nucleoside transporters), member 2 \\
\hline AA490471 & SPARCL1 & SPARC-like 1 (mast9, hevin) \\
\hline AA458849 & SPINT2 & Serine protease inhibitor, Kunitz type, 2 \\
\hline NM_152999.2 & STEAP2 & Six-transmembrane epithelial antigen of prostate 2 \\
\hline AA455272 & TMEM187 & Chromosome $\mathrm{X}$ open reading frame 12 \\
\hline AI628066 & XIAP & Baculoviral IAP repeat-containing 4 \\
\hline ВC058011 & & Hypothetical protein LOC286440 \\
\hline
\end{tabular}

$>80 \%$ ) on the growth of WI38VA13 cells. Cells treated with single-stranded control DNA and the double-stranded DNAlipid complex showed no significant inhibition of cell growth.

\section{Discussion}

The single-stranded LC-sense molecules were utilized as probes for DNA microarrays and showed stronger binding signals than those of the PCR-amplified cDNA probes under identical hybridization conditions. The stronger signals observed with the LC-sense DNA microarray can be attributed to the favorable environment for intermolecular hybridization provided by the single-stranded structural feature of LCsense DNA, coupled with its considerable length. Repeated microarray experiments using LC-sense probe DNA generated convincing results. Reliability was then confirmed using quantitative real-time RT-PCR, and fold changes obtained from microarray and real-time RT-PCR were well correlated. Large-scale LC-sense DNA microarrays were then prepared, and a dye-swap test was conducted. Result of the dye-swap test also showed good correlation coefficiency. By employing LC-antisense molecules to overexpressed genes in the $5 \mathrm{~K}$ microarray, 28 genes functionally involved in the growth of lung cancer cells were identified.

Several features of LC-sense probe DNA make it practical for gene expression profiling. First, LC-sense DNA can be generated in a large quantity and at a reasonable cost. LCsense DNA can be dependably generated from the culture supernatants of competent bacterial cells already harboring helper bacteriophages and transformed with recombinant M13 phagemids (21). Second, the single-stranded nature of LC-sense DNA allows stronger binding to target genes as compared to that of double-stranded cDNA. Third, LC-sense DNA has replication fidelity identical to that of bacterial genomic DNA. Furthermore, target validation can be easily accelerated by subsequent employment of LC-antisense libraries allowing functional analysis of genes.

Massively parallel expression profiling with DNA microarrays has recently emerged as a leading technology in the systematic analysis of cellular physiology (31). DNA micro- 
arrays are currently being used in a variety of applications, including gene discovery (32), disease diagnosis (33), drug discovery (34) and toxicological research (35). The intensity of hybridization signals on DNA microarrays is an important factor, as weak signals render the microarray data inconsistent, and limit many of the potential applications of this technology (19). Due to stronger binding signals, LC-sense microarray experiments require smaller amounts of probe DNA for the production of the DNA microarrays, less Cy3 and Cy5 dye in the generation of downstream data, and smaller amounts of RNA samples.

The acquisition of meaningful data from gene expression profiles, such as powerful diagnostic markers or novel targets for the treatment of cancer, is important but difficult. When certain genes are overexpressed in cancer cells, they may have a role in cancer or pathologic cell growth, and may be selected as potential therapeutic targets. Therefore, functional studies on an individual gene employing antisense oligonucleotides (36) or si-RNA (13-15) are necessary for each gene. In this study, we identified 28 candidate genes which inhibited the growth of A549 but not WI38VA13 cells. These 28 genes appear to have functions that are either directly or indirectly associated with the growth of lung cancer cells. Of these genes, several were already known as targets of anticancer therapy or tumor markers. XIAP is a well-known inhibitor of the apoptosis protein family, and RNA interference of XIAP for cancer therapy has been attempted (37). Specific inhibition of Lyn using siRNA significantly decreased tumor growth and also reduced metastases (38), and a recent bioinformatical study revealed that CYP24A1 is a valuable biomarker in lung cancer (39). However, further studies are required which apply these results to clinical settings, since these results were obtained in cell lines.

In conclusion, LC-sense DNA demonstrates suitable properties for microarray experiments. Furthermore, LCsense and -antisense libraries may be effectively combined for target gene validation in a high-throughput mode.

\section{Acknowledgements}

This study was supported by the Korea Science and Engineering Foundation (KOSEF) through the CDRC Center of Keimyung University (R13-2002-028-01004-0), the Korean Ministry of Commerce, Industry and Energy (10009426). This research was supported, in part, by the Korean BioInformation Center (KOBIC) Research Support Program. We especially thank Dr Sangbae Kim for the deposition of microarray data at GEO.

\section{References}

1. Schena M, Shalon D, Davis RW and Brown PO: Quantitative monitoring of gene expression patterns with a complementary DNA microarray. Science 270: 467-470, 1995.

2. Lockhart DJ, Dong H, Byrne MC, et al: Expression monitoring by hybridization to high-density oligonucleotide arrays. Nat Biotechnol 14: 1675-1680, 1996.

3. DeRisi JL, Iyer VR and Brown PO: Exploring the metabolic and genetic control of gene expression on a genomic scale. Science 278: 680-686, 1997 .

4. Iyer VR, Eisen MB, Ross DT, et al: The transcriptional program in the response of human fibroblasts to serum. Science 283: $83-87,1999$.
5. Nguyen DV, Arpat AB, Wang $\mathrm{N}$ and Carroll RJ: DNA microarray experiments: biological and technological aspects. Biometrics 58: 701-717, 2002

6. Ramaswamy S and Golub TR: DNA microarrays in clinical oncology. J Clin Oncol 20: 1932-1941, 2002.

7. Golub TR, Slonim DK, Tamayo P, et al: Molecular classification of cancer: class discovery and class prediction by gene expression monitoring. Science 286: 531-537, 1999.

8. Ji W, Zhou W, Gregg K, Yu N, Davis S and Davis S: A method for cross-species gene expression analysis with high-density oligonucleotide arrays. Nucleic Acids Res 32: e93, 2004.

9. Gottschalk AR, Basila D, Wong M, Dean NM, Brandts CH, Stokoe D and Haas-Kogan DA: p27Kip1 is required for PTEN-induced G1 growth arrest. Cancer Res 61: 2105-2111, 2001.

10. Zeng JZ, Wang HY, Chen ZJ, Ullrich A and Wu MC: Molecular cloning and characterization of a novel gene which is highly expressed in hepatocellular carcinoma. Oncogene 21: 4932-4943, 2002.

11. Ahn JD, Morishita R, Kaneda Y, et al: Inhibitory effects of novel AP-1 decoy oligodeoxynucleotides on vascular smooth muscle cell proliferation in vitro and neointimal formation in vivo. Circ Res 90: 1325-1332, 2002.

12. Suyama E, Kawasaki H, Nakajima M and Taira K: Identification of genes involved in cell invasion by using a library of randomized hybrid ribozymes. Proc Natl Acad Sci USA 100: 5616-5621, 2003

13. Paddison PJ, Silva JM, Conklin DS, et al: A resource for largescale RNA-interference-based screens in mammals. Nature 428: 427-431, 2004.

14. Berns K, Hijmans EM, Mullenders J, et al: A large-scale RNA screen in human cells identifies new components of the p53 pathway. Nature 428: 431-437, 2004.

15. Silva JM, Li MZ, Chang K, et al: Second-generation shRNA libraries covering the mouse and human genomes. Nat Genet 37: 1281-1288, 2005.

16. Caplen NJ: RNAi as a gene therapy approach. Expert Opin Biol Ther 3: 575-586, 2003.

17. Kittler R, Pelletier L, Heninger AK, et al: Genome-scale RNAi profiling of cell division in human tissue culture cells. Nat Cell Biol 9: 1401-1412, 2007.

18. Lee JK, Bussey KJ, Gwadry FG, et al: Comparing cDNA and oligonucleotide array data: concordance of gene expression across platforms for the NCI-60 cancer cells. Genome Biol 4 R82, 2003.

19. Asyali MH, Shoukri MM, Demirkaya O and Khabar KS: Assessment of reliability of microarray data and estimation of signal thresholds using mixture modeling. Nucleic Acids Res 32: 2323-2335, 2004

20. Lee YH, Moon IJ, Hur B, et al: Gene knockdown by large circular antisense for high-throughput functional genomics. Nat Biotechnol 23: 591-599, 2005.

21. Jupin I and Gronenborn B: Abundant, easy and reproducible production of single-stranded DNA from phagemids using helper phage-infected competent cells. Nucleic Acids Res 23: $535-536,1995$.

22. Ledakis P, Tester WT, Rosenberg N, Romero-Fischmann D, Daskal I and Lah TT: Cathepsins D, B, and L in malignant human lung tissue. Clin Cancer Res 2: 561-568, 1996

23. Busto R, Prieto JC, Bodega G, Zapatero J, Fogue L and Carrero I: VIP and PACAP receptors coupled to adenylyl cyclase in human lung cancer: a study in biopsy specimens. Peptides 24: 429-436, 2003.

24. Taichman DB, Loomes KM, Schachtner SK, et al: Notch1 and Jagged 1 expression by the developing pulmonary vasculature. Dev Dyn 225: 166-175, 2002.

25. Fukunaga Y, Bandoh S, Fujita J, et al: Expression of cytokeratin 8 in lung cancer cell lines and measurement of serum cytokeratin 8 in lung cancer patients. Lung Cancer 38: 31-38, 2002.

26. Lakka SS, Konduri SD, Mohanam S, Nicolson GL and Rao JS: In vitro modulation of human lung cancer cell line invasiveness by antisense cDNA of tissue factor pathway inhibitor-2. Clin Exp Metastasis 18: 239-244, 2000.

27. Chang CC, Shih JY, Jeng YM, et al: Connective tissue growth factor and its role in lung adenocarcinoma invasion and metastasis. J Natl Cancer Inst 96: 364-375, 2004

28. Sathyanarayana UG, Toyooka S, Padar A, Takahashi T, Brambilla E, Minna JD and Gazdar AF: Epigenetic inactivation of laminin-5-encoding genes in lung cancers. Clin Cancer Res 9: 2665-2672, 2003. 
29. Nagayama M, Sato A, Hayakawa H, Urano T, Takada Y and Takada A: Plasminogen activators and their inhibitors in nonsmall cell lung cancer. Low content of type 2 plasminogen activator inhibitor associated with tumor dissemination. Cancer 73: 1398-1405, 1994.

30. Zhou R and Skalli O: Identification of cadherin-11 down-regulation as a common response of astrocytoma cells to transforming growth factor-alpha. Differentiation 66: 165-172, 2000.

31. Young RA: Biomedical discovery with DNA arrays. Cell 102: 9-15, 2000

32. Porkka KP and Visakorpi T: Detection of differentially expressed genes in prostate cancer by combining suppression subtractive hybridization and cDNA library array. J Pathol 193: 73-79, 2001.

33. Alizadeh AA, Eisen MB, Davis RE, et al: Distinct types of diffuse large B-cell lymphoma identified by gene expression profiling. Nature 403: 503-511, 2000.

34. Shi LM, Fan Y, Lee JK, et al: Mining and visualizing large anticancer drug discovery databases. J Chem Inf Comput Sci 40: 367-379, 2000 .
35. Nuwaysir EF, Bittner M, Trent J, Barrett JC and Afshari CA: Microarrays and toxicology: the advent of toxicogenomics. Mol Carcinog 24: 153-159, 1999

36. Yonekura H, Migita H, Sakurai S, et al: Antisense display - a method for functional gene screening: evaluation in a cell-free system and isolation of angiogenesis-related genes. Nucleic Acids Res 27: 2591-2600, 1999.

37. Kunze D, Wuttig D, Fuessel S, et al: Multitarget siRNA inhibition of antiapoptotic genes (XIAP, BCL2, BCL-X(L)) in bladder cancer cells. Anticancer Res 28: 2259-2263, 2008.

38. Guan H, Zhou Z, Gallick GE, et al: Targeting Lyn inhibits tumor growth and metastasis in Ewing's sarcoma. Mol Cancer Ther 7: 1807-1816, 2008

39. Kim B, Lee HJ, Choi HY, et al: Clinical validity of the lung cancer biomarkers identified by bioinformatics analysis of public expression data. Cancer Res 67: 7431-7438, 2007. 